\title{
DSP-Based Fuzzy Control of A Multifingered Robot Hand
}

\author{
Li-Ren Lin * and Han-Pang Huang ** \\ Robotics Laboratory, Department of Mechanical Engineering \\ National Taiwan University, Taipei, TAIWAN 10674, R.O.C. \\ TEL/FAX: (886)2-3633875 \\ e-mail: hphuang@w3.me.ntu.edu.tw \\ * Professor and correspondence addressee \\ * Graduate student
}

\begin{abstract}
This paper presents the fuzzy control of a multifingered robot hand by using the digital signal processor (DSP). Since there are five fingers and seventeen joints to be controlled simultaneously, a special designed control system with fuzzy controller is developed to deal with the nonlinear behavior and large computation load of the multifingered robot hand. The fuzzy controller dealing with the level of finger control, is a multiple-input-multiple-output (MIMO) fuzzy leaming controller and is implemented in DSP chip. Furthermore, the communication function of the control system is designed so that the knowledge bases can be loaded for high level computation and modified during run time.
\end{abstract}

\section{INTRODUCTION}

The control of the multifingered robot hand is very complicated [11]. The reason is that the degrees of freedom (DOF) of the multifingered robot hand are too many and the dynamics of the hand are highly nonlinear and coupled. Once the tactile sensors are introduced, it becomes a much more complex control problem that deals with both position and force.

In literature, many researches deal with the problem of position and force control. A typical approach is the hybrid position/force control [10]. In this method, the control problem is divided into a set of position and force constraints that depend on the mechanical and geometrical features of the task to be performed. In general, this control scheme performs well for simple surface geometry, but it needs more modifications and advanced strategies to handle complex surfaces. The impedance control is designed to track the relation between the velocity of the manipulator and the interaction forces in order to generate compliant motion [3]. This method is not appropriate for the application requiring a desired contact force trajectory and results in oscillatory motion due to the introduced environmental stiffness.

Since Zadeh's paper on fuzzy set [13], fuzzy control has become one of the most active and fruitful areas in the applications of fuzzy set theory. Most fuzzy logic control relies on the operators' experiences to design the knowledge bases since the pioneering work of Mamdani [8]. The adaptive fuzzy system equipped with a training algorithm is also applied to the control problem $[5,9,12]$. Due to the self-organized mechanism, the adaptive fuzzy control can resolve system uncertainty with less heuristic information. In the research of Bekey [1], a knowledge-based planner was proposed to select grasping postures by reasoning from symbolic information of the target object geometry and the nature of the task. However, the knowledge about grasping in the sense of control was not discussed.

In this paper, we deal with the grasp control of the multifingered robot hand. An adaptive fuzzy control algorithm is proposed to carry out the simultaneous control of both the force and position. The linguistic knowledge bases of grasp are embedded with the fuzzy control. The fuzzy approximation is also introduced for the self-organized mechanism to improve the performance of grasping. The adaptive fuzzy controller is further implemented in DSP chip and gives satisfactory results.

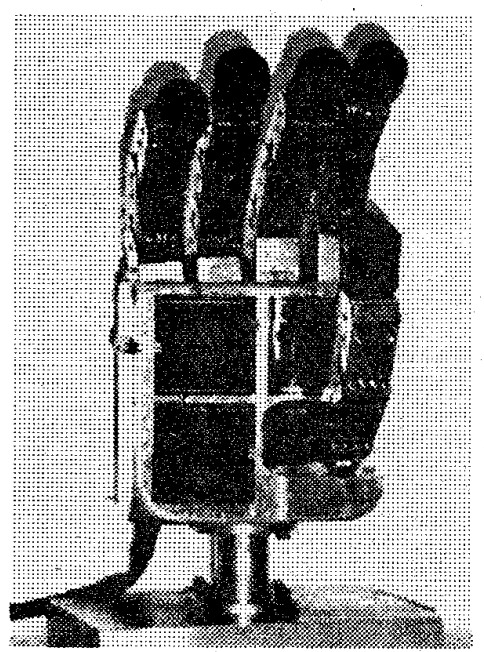

Figure 1. The NTU hand 


\section{THE NTU HAND AND ITS CONTROL SYSTEM}

The multifingered robot hand we use is the NTU hand, which is designed and fabricated in our laboratory and aims to achieve dexterous manipulation $[4,7]$. The NTU hand has five fingers with seventeen degrees of freedom (DOF). Both thumb and the first finger have four joints; two at knuckle, one between proximal and middle finger segments, and one between middle and distal finger segments. Other fingers have three joints; but only one at knuckle. Each finger is equipped with tactile sensors to detect grasping force. The tactile sensors of the hand are attached to the inner sides of finger segments and the palm to detect the contact force, as shown in Fig.1.

We provide an control architecture with a special designed hardware to control seventeen finger joints of the NTU hand. The control system, as shown in Fig.2, consists of host computer, digital central controller and several analog control modules. The host computer is a SUN workstation, which uses a three-dimensional graphic front-end to monitor the posture of the NTU hand while the position changes. It primarily performs the coordination planning of dextrous manipulation, high level computation of control and the modification of knowledge bases for grasping.

The digital central controller contains an ADSP 2101-44 DSP chip and peripheral devices to perform the fuzzy control in terms of sensor data and commands from the host computer, then distributes commands to each analog control module, and thus sends information to the host workstation. The analog controller is a PD (proportional plus derivative) controller with power amplifier. It performs the joint space control for each finger joint and shares the computation load.

\section{THE CONTROL SCHEME}

The control scheme of the NTU hand can be divided into three major parts. The first part is the knowledge bases of grasping experiences, which is the source of the control strategy. The second part is the MIMO fuzzy controller, which performs the fuzzy logic inference from the knowledge bases to exert the grasp control plus position and force. The third part is the self-organized mechanism in order to improve performance, which modified the knowledge bases from the relationship of the inputs and outputs.

\subsection{Knowledge Bases of the Furzy Control}

By observing the grasping of the human hand, the initial stage is to make in touch with the object. Once the object is in touch, the fingers exert force to hold the object. In the meantime, the posture of the hand is adjusted to meet the desired task. The grasping process is shown in Fig.3. Since the human being is an excellent expert for grasping, the amount of force applied by the fingers and the posture change are smoothly coordinated. However, the knowledge of experienced

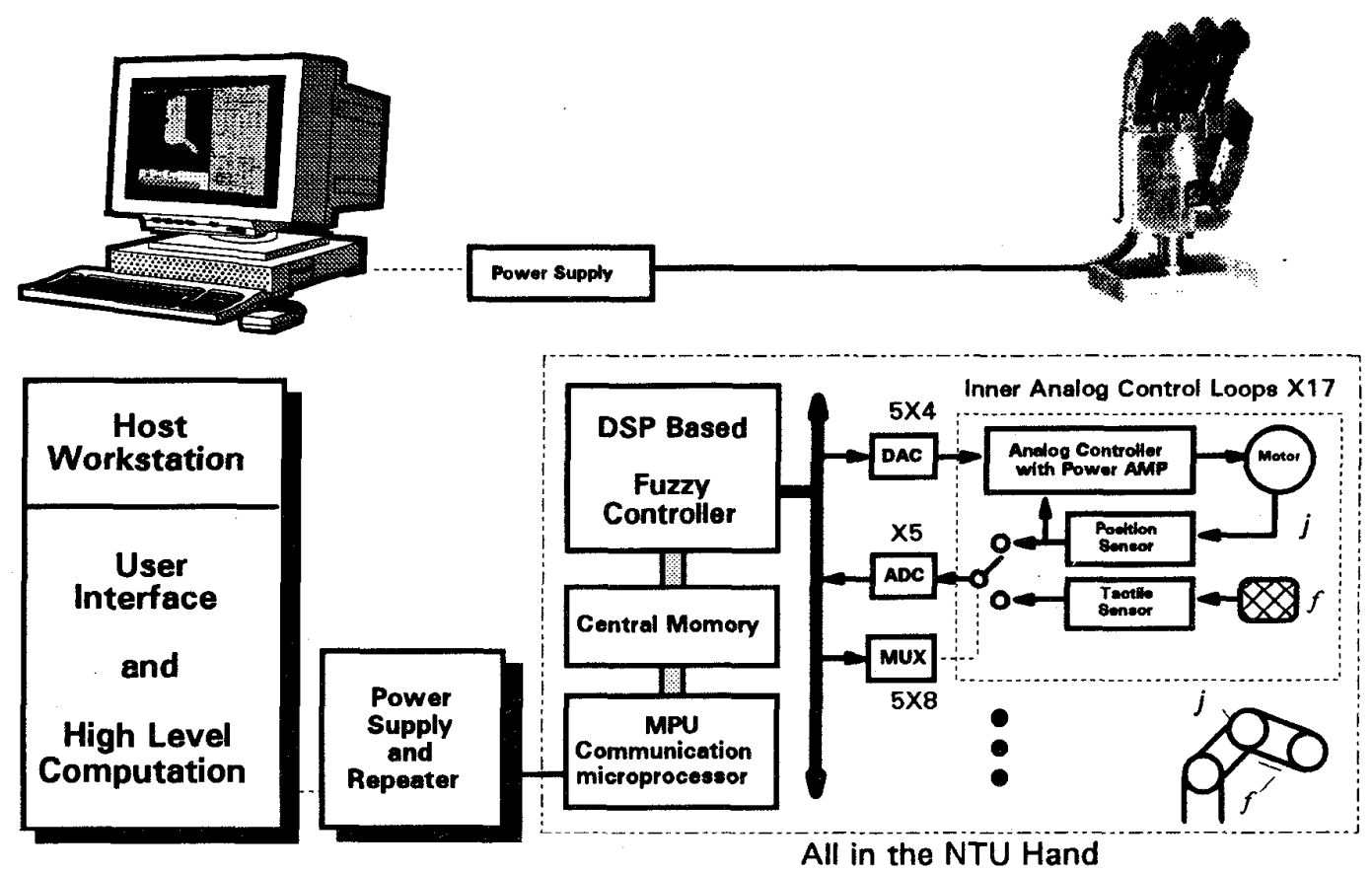

Figure 2. The control system of the NTU hand 
grasping is important for the control of the NTU hand.

In this paper, the fuzzy control is introduced to embed the linguistic experience of human. In the knowledge bases of the system, some rules and membership function are designed to accommodate the backlash and friction of gear transmission.

\subsection{MIMO Fuzzy Logic Controller}

The fuzzy controller of the control system is shown in Fig.4. The controller for each finger contains six inputs and three outputs. The six inputs consist of three joint positions and three tactile forces, while the three outputs are from analog controllers.

The fuzzy rule bases consist of a collection of fuzzy IFTHEN rules in the form:

$$
\begin{gathered}
R_{\text {vamo }}^{\prime}: \text { IF } x_{1} \text { is } F_{1}^{l} \text { and } \cdots \text { and } x_{n} \text { is } F_{n}^{l} \text { THEN } \\
y_{1} \text { is } G_{1}^{\prime} \text { and } \cdots \text { and } \mathrm{y}_{m} \text { is } G_{m}^{l}
\end{gathered}
$$

where $F_{i}^{l}$ and $G_{i}^{l}$ are fuzzy sets in $U_{i} \subset R$ and $V_{i} \subset R$, respectively, and $\underline{x}=\left(x_{1}, \cdots, x_{n}\right) \in U_{1} \times \cdots \times U_{n}$ and $y=\left(y_{1}, \cdots\right.$, $\left.y_{m}\right) \in V_{l} \times \cdots \times V_{m}$ are linguistic variables. Let $p$ be the number of fuzzy rules (i.e. $l=1,2, \ldots, p$ ) and $\underline{x}$ and $y$ be the input and output of the system. The fuzzy rules can be organized as [6]:

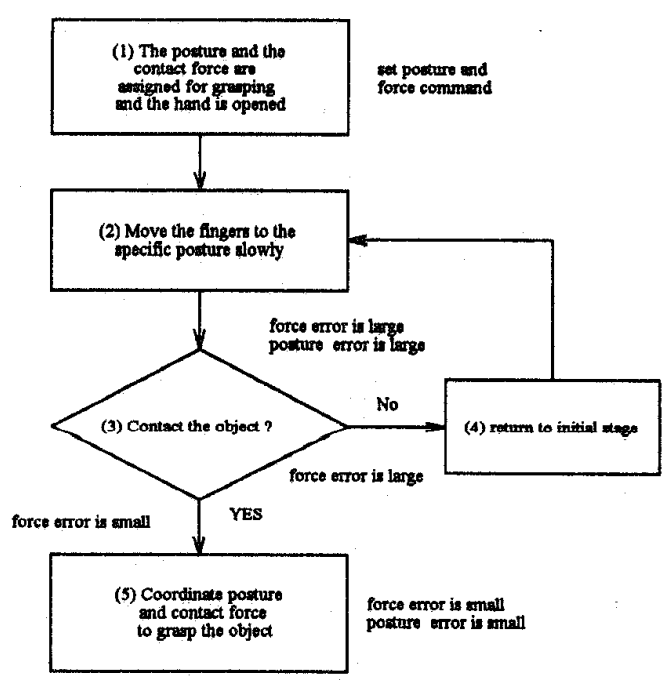

Figure 3. The grasping experience of human being

$$
\begin{aligned}
& R_{\text {anos }}=\left\{\bigcup_{l=1}^{p} R_{\text {maxs }}^{\prime}\right\} \\
& =\left\{\bigcup_{l=1}^{p}\left[\left(F_{1}^{\prime} \times \cdots \times F_{n}^{\prime}\right) \rightarrow\left(y_{1}+\cdots+y_{m}\right)\right]\right\} \\
& =\left\{\bigcup_{k=1}^{m} \bigcup_{l=1}^{p}\left[\left(F_{1}^{\prime} \times \cdots \times F_{k}^{l}\right) \rightarrow y_{k}\right]\right\}
\end{aligned}
$$

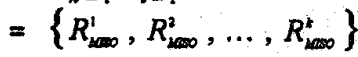

This shows that the general rule structure of a MMO fuzzy system can therefore be represented by a collection of multiple-input-single-output (MISO) fuzzy system.

According to Eq.(2), the divided MISO fuzzy system can be used for the inference of control. The fuzzy logic systems with center average defuzzifier, product-inference rule and singleton fuzzifier are of the following form [12]:

$$
y_{k}^{c}\left(x_{1}, \ldots, x_{n}\right)=\frac{\sum_{i=1}^{p} \xi_{k}\left(\prod_{i=1}^{n} \mu_{B_{l}}\left(x_{i}\right)\right)}{\sum_{l=1}^{p}\left(\prod_{i=1}^{n} \mu_{p_{l}}\left(x_{l}\right)\right)}, \quad k=1,2,3
$$

where $y_{k}{ }^{c}$ is the output of the MISO fuzzy control and $\mu_{F_{i}}$ denotes the membership function for the fuzzy set $F_{i}^{l}$. The $\xi_{k}^{l}$ is the center of the fuzzy set $G_{k}^{l}$; that is, the point in $V_{k}$ at which the membership function $\mu_{G^{\prime}}$ of $G_{k}^{l}$ achieves its maximum value.

The input $\underline{x}$ of the fuzzy logic controller is the error between the desired finger joint position/force and actual position/force. The output $y^{c}$ of the controller is the difference between the command of the analog inner loop controller and actual joint force/torque. Namely,

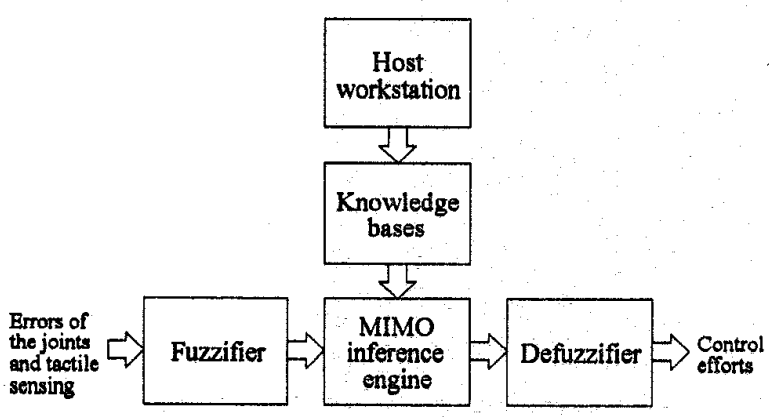

Figure 4. The fuzzy controller for one finger 


$$
\left[\begin{array}{l}
x_{1} \\
x_{2} \\
x_{3} \\
x_{4} \\
x_{5} \\
x_{6}
\end{array}\right]=\left[\begin{array}{l}
\ddot{j_{1}}-j_{1} \\
j_{2}-j_{2} \\
\dot{j_{3}}-j_{3} \\
f_{1}^{*}-f_{1} \\
f_{2}^{*}-f_{2} \\
f_{3}^{*}-f_{3}
\end{array}\right] \text { and } y^{c}=\left[\begin{array}{l}
y_{1}^{c} \\
y_{2}^{c} \\
y_{3}^{c}
\end{array}\right]=\left[\begin{array}{c}
\tau_{1}^{c} \\
\tau_{2} \\
\tau_{3}
\end{array}\right]
$$

where $j_{i}^{*}$ is the desired joint command; $f_{i}^{*}$ is the desired force command; $j_{i}$ and $f_{i}$ are the feedback of the joint and tactile sensors. The control effort $\tau_{i}^{c}$ is the joint torque of the $i$ th joint. Since $\tau_{i} \cong k_{p}^{i}\left(c_{i}-j_{i}\right)$, the input $c_{i}$ of the inner loop analog PD controller is obtained from the proportional gain $k_{p}^{i}$.

Since there are many computations in table lookup, multiplication and summation, a DSP chip is introduced to perform the fuzzy inference. The implementation of the fuzzy controller is direct coding in assembly language and takes advantage of the features of the DSP to speed up the whole system.

\subsection{Self-Organized Mechanism}

The self-organized mechanism is designed to improve the grasping performance to meet the requirements. Although the linguistic descriptions of the grasp experience of human are given, the knowledge bases and membership functions must be adjusted during simulation and experiments.

Since the computations of the training algorithm are huge and the task can be separated from the control, we provide a fuzzy development environment to distribute the computation load. The man machine interface (MMI) and graphic simulation environment are developed in the workstation, while the low level control for five fingers is based on a DSP processor. The communication between the high level MMI and the low level control is through special designed communication modules.

Each sampling period contain six sensor data and three analog commands of the inner control loop for each finger. Denote the sensor data (three joint positions and three tactile force sensings) as $\underline{x}_{s}$, and denote the three commands to inner control loop as $\underline{c}_{s}$. $\left(c_{s}, \underline{x}_{s}\right)$ forms a set of input-output training pairs. The corresponding output $y_{s}$ is obtained by the following equation

$\underline{x}_{s}=\left[\begin{array}{l}j \\ \underline{f} \\ \underline{f}\end{array}\right]=\left[\begin{array}{l}j_{s 1} \\ j_{s 2} \\ j_{s 3} \\ f_{s 1} \\ f_{s 2} \\ f_{s 3}\end{array}\right]$ and $\underline{y}_{s}=\underline{k}_{p}^{\tau}\left(c_{s}-\underline{j}_{s}\right)=\left[\begin{array}{l}k_{p}^{1}\left(c_{s 1}-j_{s 1}\right) \\ k_{p}^{2}\left(c_{s 2}-j_{s 2}\right) \\ k_{p}^{3}\left(c_{s 3}-j_{s 3}\right)\end{array}\right]$

The self-organized mechanism learns the relation between $y_{s k}$ and $f_{s k}$ for $f_{s k}>0(k=1,2,3)$. The learning algorithm is based on a fuzzy approximation. The membership function of the fuzzy approximation is the same as the fuzzy controller, and the learning algorithm is as follows:

$f_{s k}^{\prime}(\underline{y})=\frac{\sum_{i=1}^{q} \eta_{k}^{l}\left(\prod_{i=1}^{m} \mu_{G t}\left(y_{i}\right)\right)}{\sum_{i=1}^{q}\left(\prod_{i=1}^{m} \mu_{G t^{\prime}}\left(y_{i}\right)\right)}$, for $f_{s k}>0, k=1,2,3$

for the maximum firing strength of the rule of $k$-th joint

$$
\eta_{k}^{\prime}(n+1)=\eta_{k}^{\prime}(n) \frac{f_{\text {sk }}}{f_{\text {sk }}^{\prime}}, \quad n=0,1,2, \ldots
$$

where $f_{s k}^{\prime}$ is the output of the MISO fuzzy approximation and $\mu_{G_{k}^{\prime}}$ denotes the membership function of the fuzzy set $G_{k}^{l} \eta_{k}^{l}$ is the center of the fuzzy set $H_{k}^{l}$ in $W_{k} \subset R$; i.e., the point in $W_{k}$ at which the membership function $\mu_{H^{\prime}}$ of $H_{k}^{l}$ achieves its maximum value. In Eq.(6), $f_{s k}^{\prime}$ must be positive for the fact that the tactile sensing is always positive. In Eq.(7), $\eta_{k}^{l}(0)$ must not be zero initially. All the training pairs are used in the fuzzy approximation of Eqns.(6-7). The algorithm keeps learning until the errors are small enough.

Once the relation between $y_{s}$ and $f_{s}$ is learned by learning algorithm, the knowledge bases of the fuzzy approximation can be obtained. Since the fuzzy sets $H_{k}^{l}$ in Eq.(6) are not the same as the fuzzy sets $F_{i}^{l}$ in Eqns.(1-3), the results of the fuzzy approximation require slight modification in order to be applied to control strategy.

\section{THE DSP BASED FUZZY CONTROL}

The control system of the NTU hand provides a development environment for fuzzy control. Due to the use of special designed communication modules between the workstation and the digital central controller, the knowledge bases of the fuzzy control can be initially set from the simulation result in the workstation and modified during the operation. During the run time, the self-organized mechanism still works in the workstation to monitor the performance of the whole hand at each sampling interval. The user can save the improved knowledge bases for further use or down load into the controller immediately.

The whole finger hand is controlled by the MMO fuzzy controller. Each finger of the NTU hand can be treated as a linked robot. Since there are five fingers with seventeen joints to be controlled simultaneously, there will be five fuzzy controllers to be computed in the same time in order to control the NTU hand. The computation load should be shared by other 
device other than the central controller to perform low level control. By introducing the inner loop analog PD control, the sampling rate of the DSP fuzzy controllers is reduced an order.

In order to get efficient inference of the fuzzy control, the assembly language is used to code DSP programs. From the technical reference of the DSP [2], the computation can be speeded up by using convolution calculation (summation of multiplication). That is why we use the product-inference in Eq.(3). In the DSP fuzzy control, up to $4 \times 10^{5}$ rules can be inferred in one second. The major limitation of speed is the conversion time of the analog to digital converter. The sampling period of each joint control is $10 \mathrm{~ms}$ in the DSP controller. However, parts of the computation are spent on the defuzzifier process. A more efficient method of defuzzifier will speed up the computation.

\section{RESULTS OF SIMULATION AND EXPERIMENTS}

Parts of the results of the simulation and experiments are shown in the Fig.5-8. In general, the difference between the simulation and experiment in the joint control case is larger than the force control case. The reason is that the self-organized mechanisms in the development environment of the NTU hand only deal with the relation of position and force. The MMI of the fuzzy development environment is shown in Fig.9. The MMI shows the signals of the control during simulation and experiments. The parameters of the controller can be adjusted from the control panel during operations. The three dimensional graphics are used to show the posture, and the gray color is used to show the intensity of the tactile force on the NTU hand.

\section{CONCLUSIONS}

In this paper, a DSP based fuzzy control of the multifingered robot hand is developed. Since there are so many joints to be controlled simultaneously and involve hand dynamics of movement and force, the adaptive fuzzy control is implemented to take the features of the DSP, and a hierarchical architecture of the control system is proposed to arrange the computation load. A fuzzy development environment is also built to show the signals of the simulation and experiment, and the three dimensional graphics are used to show the posture and status of the NTU hand. Furthermore, according to communication functions of the control system, the knowledge bases can be loaded for high level computation and modified during run time.

\section{ACKNOWLEDGMENT}

This research is partially supported by National Science Council of R.O.C. under Grant number NSC 83-0422-E002093.

\section{REFERENCES}

[1] G.A. Bekey, H. Liu, R. Tomovic and W.J. Karplus, "Knowledge-Based Control of Grasping in Robot Hands Using Heuristics from Human Motor Skills," IEEE Transactions on Robotics and Automation, Vol. 9, No. 6, pp. 709-722, 1993.

[2] DSP Division, ADSP-2101/2102 User's Manual, Analog Devices Inc., U.S.A., 1991.

[3] N. Hogan, "Stable Execution of Contact Tasks Using Impedance Control," IEEE Conference on Robotics and Automation, pp. 1047-1054, 1987.

[4] H.P. Huang and L.R. Lin, "Development of A Prosthetic Hand with Multifingers," NSC Report of R.O.C., NSC 830422-E002-093.

[5] J.S. Jang, "Self-Learning Fuzzy Controllers Based on Temporal Back Propagation;" IEEE Transactions on Neural Network, Vol.3, pp. 723-741, 1992.

[6] C.F. Lin, Advanced Control System Design, PrenticeHall, New Jersey, pp.441-446, 1994.

[7] L.R. Lin and H.P. Huang, "Use Hopfield Networks to Find A Grasping Solution for A Dextrous Hand," IEEE Intermational Symposium on Artificial Neural Networks, 1994.

[8] E.H. Mamdami and S. Assilian, "An Experiment in Linguistic Synthesis with a fuzzy logic controller," International Joumal of Man-Machine Studies, Vol. 8, pp. 249-291, 1976.

[9] D. Nauck and R. Kruse, "A Fuzzy Neural Network Learning Fuzzy Control Rules and Membership Functions by Fuzzy Error Backpropagation," Proceeding of IEEE Intemational Conference on Neural Networks, pp. 1022-1027, 1993.

[10] M.H. Raibert and J.J. Craig, "Hybrid Position/Force Control of Manipulators," ASME Journal of Dynamic Systems, Measurement, and Control, Vol. 102, pp. 126-133, June 1981.

[11] T.H. Speeter, "Control of the Utah/MIT Dextrous Hand: Hardware and Software Hierarchy," Journal of Robotic Systems, Vol.7, No.5, pp. 759-790, 1990.

[12] L.X. Wang, Adaptive Fuzzy Systems and Control: Design and Stability Analysis, Prentice-Hall, New Jersey, 1994.

[13] L.A. Zadeh, "Fuzzy Sets," Information and Control, Vol. 8, pp. 338-353, 1965. 


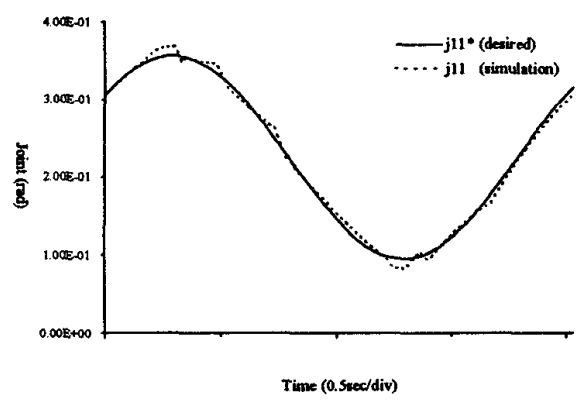

Figure 5. The simulation of joint control (finger 1,joint 1)

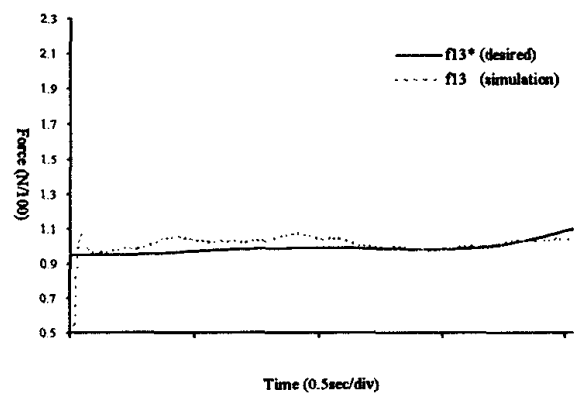

Figure 7. The simulation of force control (finger 1, joint 3)

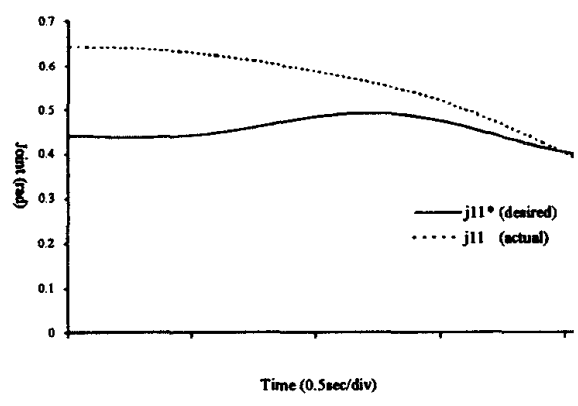

Figure 6. The experiment of joint control (finger 1, joint 1)

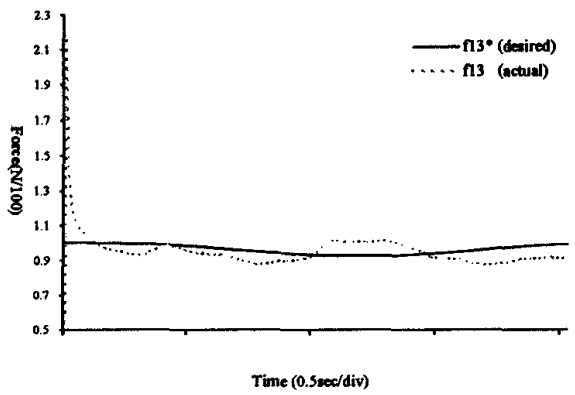

Figure 8. The experiment of force control (finger 1, joint 3)
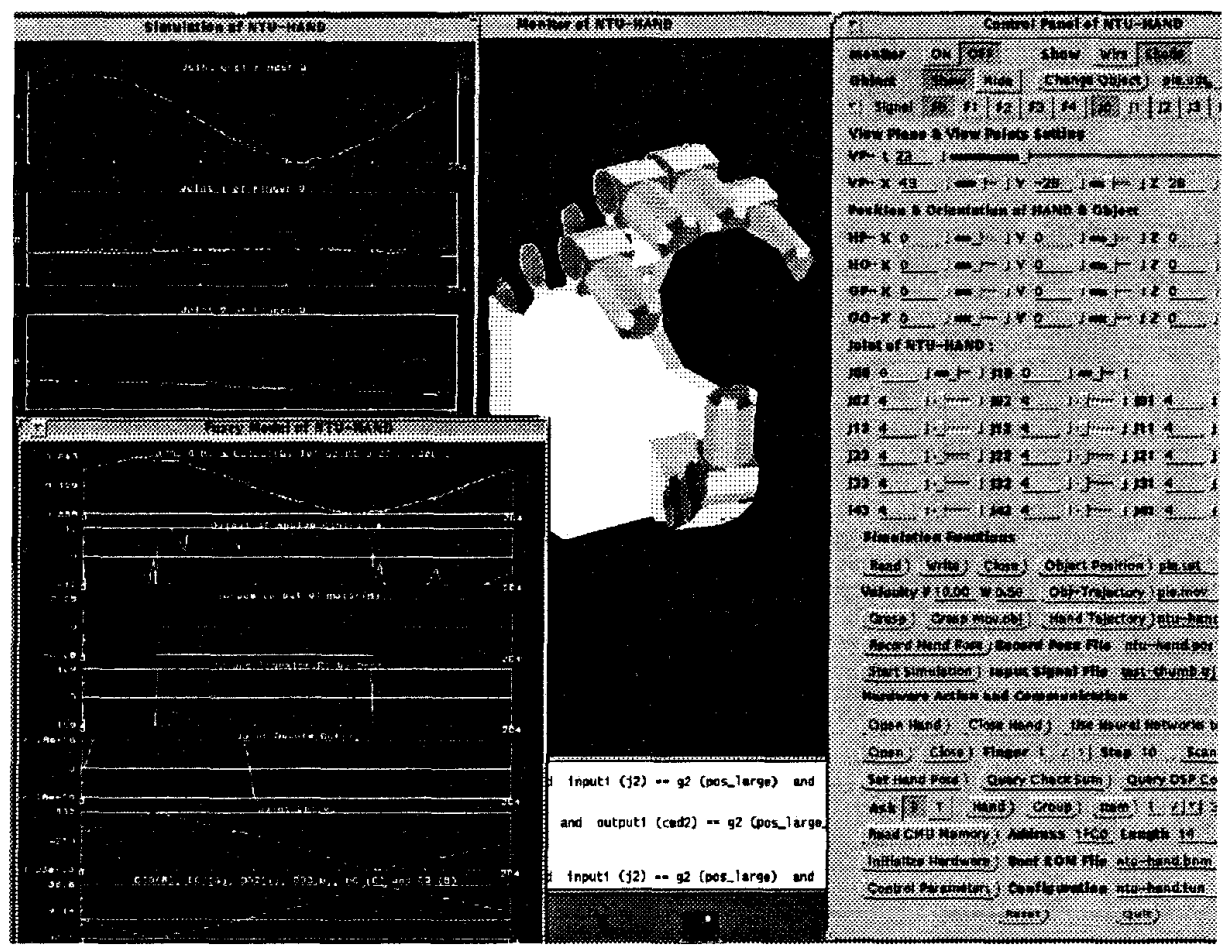

Figure 9. The user interface of the fuzzy development environment 